\title{
Magnetization switching of submicrometer Co dots induced by a magnetic force microscope tip
}

\author{
M. Kleiber, F. Kümmerlen, M. Löhndorf, and A. Wadas \\ Institute of Applied Physics and Microstructure Research Center, University of Hamburg, Jungiusstrasse 11, \\ D-20355 Hamburg, Germany \\ D. Weiss \\ Institute for Experimental and Applied Physics, University of Regensburg, D-93040 Regensburg, Germany \\ R. Wiesendanger \\ Institute of Applied Physics and Microstructure Research Center, University of Hamburg, Jungiusstrasse 11, \\ D-20355 Hamburg, Germany
}

(Received 23 September 1997; revised manuscript received 31 March 1998)

\begin{abstract}
We have applied magnetic force microscopy (MFM) with an in situ electromagnet to study the switching of the magnetization of submicrometer Co dots fabricated by means of electron-beam lithography. By using the MFM tip as a local-field source, the magnetization of individual single-domain Co dots could be reversed. Micromagnetic simulations show that the switching process is induced by the stray field of the MFM tip. Furthermore, the external field that is necessary to support switching of the dot depends on the tip-dot separation. [S0163-1829(98)01333-2]
\end{abstract}

\section{INTRODUCTION}

Nanostructured ferromagnetic particle arrays have become of increased interest because of their possible application as a high-density magnetic storage media ${ }^{1,2}$ and their potential as semiconductor-ferromagnet hybrid systems. ${ }^{3,4}$ The storage density of thin magnetic film recording media is, among other reasons, limited by domain walls and ripple structures that contribute to noise in the readback signal. ${ }^{5} \mathrm{By}$ using single-domain ferromagnetic particles as a storage media, this limitation can be overcome. Theory suggests that ferromagnetic particles below the critical size are in a single domain state. ${ }^{6,7}$ Magnetization of a single-domain particle can be in two discrete states. Therefore, each particle is able to code a single bit in a microstructured recording media. ${ }^{1}$

To study the average magnetic switching behavior of such single-domain particles, hysteresis curves of large arrays of particles have been taken using a magnetometer. ${ }^{2,8}$ Magnetic force microscopy ${ }^{9}$ (MFM) provides an excellent opportunity to image the magnetization of individual dots because of its high resolution and sensitivity. ${ }^{10,11}$

We report on an investigation of the micromagnetic properties of Co dot arrays on GaAs substrates in the presence of an external magnetic field. The experimental results are complemented by micromagnetic simulations.

\section{EXPERIMENT}

Large arrays of ferromagnetic Co dots were fabricated on GaAs substrates using electron-beam lithography. ${ }^{3,4}$ In order to improve adhesion, the GaAs substrates were covered with a Cr layer of $3 \mathrm{~nm}$ thickness.

The structures were written by an electron beam into two layers of a photoresistant material. After developing, the exposed PMMA (polymethylmetacrylate) resistant Cobalt was evaporated. After liftoff in acetone, dot arrays were obtained. The dots have diameters in the range of 100-300 $\mathrm{nm}$ and a thickness of 4-17 nm. They were studied with MFM using a
Nanoscope IIIa system ${ }^{12}$ in the dynamic "lift mode." We used Si cantilevers with integrated tips and spring constants between 3 and $5 \mathrm{~N} / \mathrm{m}$. The resonance frequencies were found to be $55-73 \mathrm{kHz}$. A single layer of $28 \mathrm{~nm}$ iron served as a magnetic thin-film coating for the tips. Magnetic force gradients were measured by detecting phase shifts in the cantilever oscillation due to attractive or repulsive forces acting on the ferromagnetic MFM tip. We added an electromagnet for applying fields in the plane of the sample. The microscope was placed in the center of a Helmholtz coil pair. In this manner we were able to apply fields up to $60 \mathrm{kA} / \mathrm{m}$.

In order to study the switching behavior, we used the following procedure. The scan area is chosen so that only the dot we want to switch the magnetization on is scanned. We use the same parameters as in all other measurements presented here. After an image of the initial state is obtained, the external field is switched on while the scanning is continued. In this way the dot is influenced by the tip's stray field and the external field at the same time. The external field is increased until the magnetization direction of the dot changes. Then the external field is turned off. The dot is scanned again to see if the magnetization remains stable without the external field.

\section{RESULTS AND DISCUSSION}

Figure 1 shows the topography of $3 \mu \mathrm{m} \times 3 \mu \mathrm{m}$ area and the corresponding MFM image of a Co dot array with a height of $7 \mathrm{~nm}$. The dot diameter is $180 \mathrm{~nm}$ as determined from topographic measurements. The MFM image is characterized by a bright and dark contrast over each Co dot. The MFM tip was magnetized in the $z$ direction (perpendicular to the plane of the sample) prior to the measurement. Therefore, the magnetic contrast can be explained by an interaction between the in-plane magnetized dots and the MFM tip. For a Co dot height of $7 \mathrm{~nm}$ in an as-prepared state with a circular shape, we did not observe any preferential alignment of the dots' magnetization direction. 


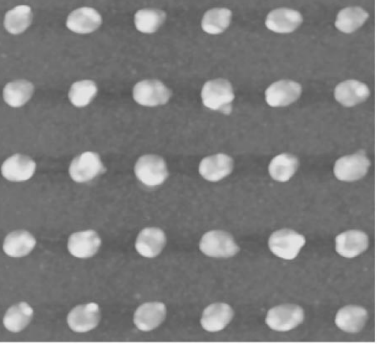

(a)

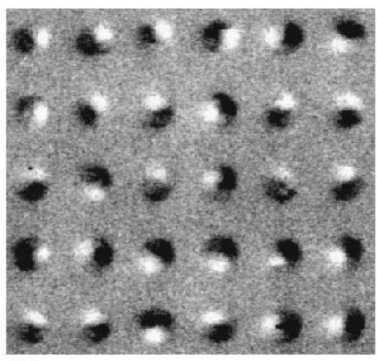

(b)
FIG. 1. (a) Topographical and (b) MFM image of circular cobalt dots (diameter: $150 \mathrm{~nm}$, thickness: $7 \mathrm{~nm}$ ) in the as-prepared state. The magnetic dipole contrast indicates that the dots are magnetized in plane. Scan size: $2 \mu \mathrm{m} \times 2 \mu \mathrm{m}$.

Changing the geometry of the dots led to a change of the magnetization direction. For elliptically shaped Co dots the magnetization direction is aligned parallel to the long axis, even for an as-prepared sample. Figure 2 presents the topography and the corresponding MFM image of an as-prepared sample with an elliptical shape of Co dots. The lateral dimensions of these dots are $140 \mathrm{~nm} \times 250 \mathrm{~nm}$ and the height is $7 \mathrm{~nm}$. The magnetization directions of the dots are aligned parallel to the long axis ( $x$ direction). Only a few dots revealed a different orientation of the magnetization.

In order to explain the magnetic contrast mechanism, we magnetized the Co dot sample in a strong external field (400 $\mathrm{kA} / \mathrm{m}$ ) outside the MFM system. The magnetic field was applied in-plane of the sample, parallel and antiparallel to the $x$ direction. Figure 3(a) shows a MFM image $(1.1 \mu \mathrm{m}$ $\times 1.1 \mu \mathrm{m}$ ) with Co dots in the remanent state. The dot array was magnetized by a field applied along the $(-x)$ direction [marked by a black arrow below Fig. 3(a)] prior to the MFM measurement. The magnetization directions of the dots are aligned in the direction of the applied field. Bright contrast on the right-hand side and black contrast on the left-hand side are clearly visible. Applying the field in the opposite $x$ direction [marked by a black arrow below Fig. 3(b)] led to an inverse magnetic contrast.

The MFM measurements were performed in the dynamic mode with a phase detection system. Resonance frequency shifts towards higher frequencies led to a bright contrast in the phase detection. Therefore, a repulsive interaction between tip and sample is represented by bright contrast and an

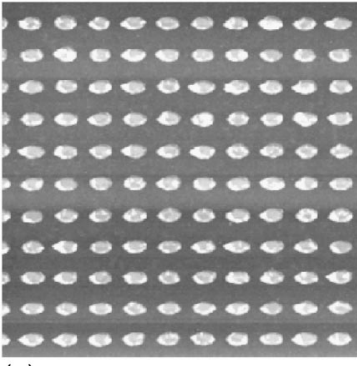

(a)

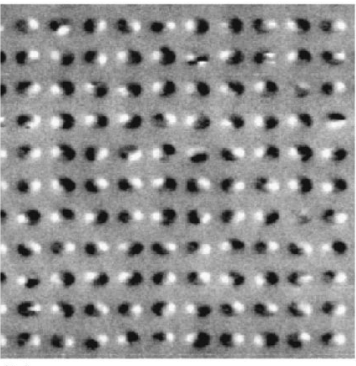

(b)

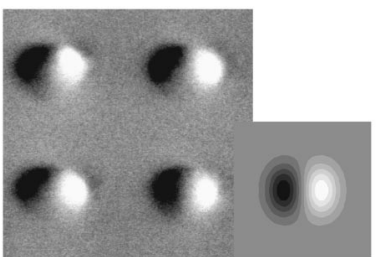

(a)

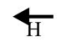

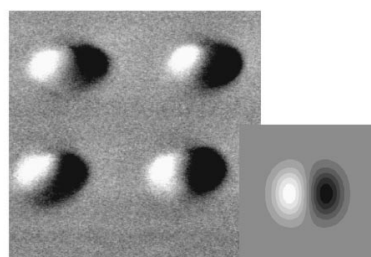

(b)
FIG. 3. MFM images of dots (diameter: $200 \mathrm{~nm}$, thickness: 17 $\mathrm{nm})$ that have been magnetized in a strong external field (400 $\mathrm{kA} / \mathrm{m}$ ) outside the microscope. After scanning (a) the field has been applied in the opposite direction. The small figures show the results of a simulation for the MFM contrast of dots magnetized in the $(x)$ and $(-x)$ direction (marked) and scanned by using a tip magnetized in the $z$ direction (perpendicular to the plane of the sample). Scan size: $1.1 \mu \mathrm{m} \times 1.1 \mu \mathrm{m}$.

attractive interaction by dark contrast. Repeating the same experiment with an in-plane magnetized MFM tip led to a magnetic contrast that is characterized by an attractive interaction above the center of a dot and a repulsive interaction at both ends [Fig. 4(a)]. After the Co dot sample was magnetized in the opposite direction, a repulsive interaction above the center of a dot and an attractive interaction at both ends was observed [Fig. 4(b)]. In conclusion, the remanent state of the Co dots could be described as a single domain. Applying an external field led to a preferential alignment of the magnetization direction of the Co dots. In order to study the magnetic switching behavior of individual dots, we placed the microscope between a pair of Helmholtz coils. We could apply a field up to $60 \mathrm{kA} / \mathrm{m}$ in-plane of the sample.

The principle of this switching process is illustrated in Fig. 5. The applied field is chosen to be not so strong as to change the magnetization of the dots. However, the dots are also influenced by the stray field of the tip. The magnetic coating of the tip is chosen in a way that its stray field does not switch the dot either. But if a dot is influenced by the superposition of the tip's stray field and the applied field, its magnetization direction should change.

In a previous experiment ${ }^{10}$ we have found that performing switching experiments with arrays of circular-shaped Co dots of $7 \mathrm{~nm}$ thickness did not lead to a change of magnetization direction by $180^{\circ}$ of individual dots. The angle between the initial and final direction of the dots' magnetization depends therefore on the shape and magnetocrystalline anisotropy of the sample.

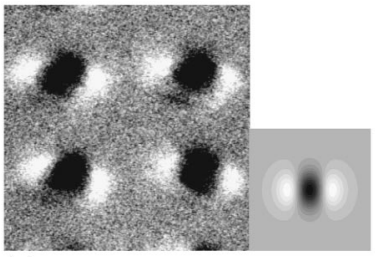

(a)

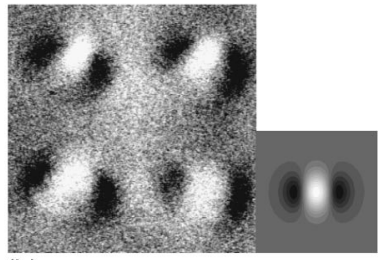

(b)
FIG. 2. (a) Topographical and (b) MFM image of elliptical cobalt dots (140 $\mathrm{nm} \times 250 \mathrm{~nm}, 7 \mathrm{~nm}$ thickness) in the as-prepared state. The magnetization of most dots is parallel to their long axes. Scan size: $6 \mu \mathrm{m} \times 6 \quad \mu \mathrm{m}$.
FIG. 4. MFM images of the dots used in Fig. 3 magnetized again in two opposite directions and scanned with a tip magnetized in the $x$ direction. Attached are the results of the simulation for a tip magnetized in the $x$ direction. Scan size: $1.1 \mu \mathrm{m} \times 1.1 \mu \mathrm{m}$. 
(a)

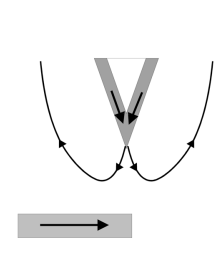

(b)

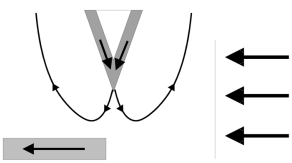

FIG. 5. Principle for magnetic switching of individual dots. The tip and the external field are chosen in a way that neither the stray field of the tip (a) nor the external field (b) are strong enough to cause a dot to switch. Only the superposition of the two leads to a change of the dot's magnetization (c).

As the elliptical dots shown in Fig. 2 are preferably magnetized in two opposite directions, they are good candidates for switching experiments. Applying an external field parallel to the long axis of the sample with a strength of $400 \mathrm{kA} / \mathrm{m}$ led to a homogeneous alignment of all Co dots. For the local MFM switching experiment, we chose an array of $3 \times 3$ dots. Figure 6 shows that the dots are all aligned in the initial state. Then the scan size is decreased so that only the dot in the middle is inside the scanned area. While the dot is scanned, the external field is increased until the magnetization direction of the dot changes. Another scan of all 9 dots taken without an external field shows that the new magnetization state of the dot in the middle is stable and that the magnetization of the other dots has not changed. In the same way,

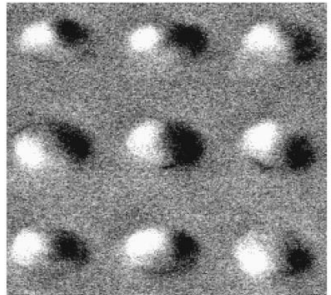

(a)

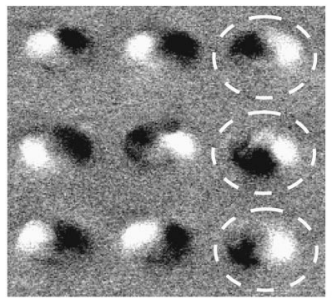

(c)

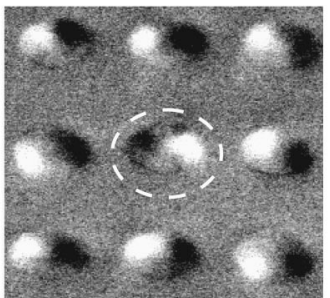

(b)

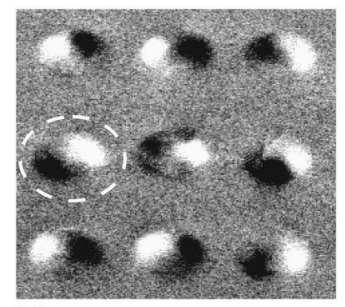

(d)
FIG. 6. Local manipulation experiment. After the dots were magnetized in a strong external field (a) was scanned. Then the dot in the middle was scanned individually with a field of $20 \mathrm{kA} / \mathrm{m}$ applied. (b) shows that this dot has switched. In the same way, each of the three dots on the right-hand side has been scanned and switched individually with fields of $22 \mathrm{kA} / \mathrm{m}$ up to $40 \mathrm{kA} / \mathrm{m}$. Finally, the dot on the left-hand side (d) was switched in the same way. we switched the magnetization of each of the three dots on the right before scanning Fig. 6(c). Finally, the dot on the left-hand side as marked in Fig. 6(d) is switched. For each dot a different external field was needed to change its magnetization. For the five switched dots, we used fields from 22 $\mathrm{kA} / \mathrm{m}$ up to $40 \mathrm{kA} / \mathrm{m}$. Other dots showed no change in their magnetization, even at fields up to $60 \mathrm{kA} / \mathrm{m}$.

The different switching behavior of the Co dots can be explained by their polycrystalline structure. ${ }^{11}$ The net magnetization of a Co dot is given by the sum of the magnetization of randomly orientated crystallites. The switching fields are also dependent on the orientation and the magnetic coupling of the crystallites.

\section{MICROMAGNETIC MODEL}

In order to investigate the switching mechanism of the Co dots in more detail, a micromagnetic simulation ${ }^{13}$ was performed. The model consists of a tip that is positioned above a single elliptical Co dot. A rectangular coordinate system $x y z$ is defined with the origin in the center of the dot and the $x$ and $y$ axis pointing along the long and short axis of the ellipse, respectively. The tip is positioned at a height $h_{t}$ above the dot and symmetrically with respect to the long axis of the dot. The tip is modeled as a nonmagnetic $\mathrm{Si}_{3} \mathrm{~N}_{4}$ body coated with $28 \mathrm{~nm}$ Fe with a homogeneous magnetization $M_{t}$ pointing in the $(-z)$ direction. The tip's magnetization was chosen to be rigid, as our experiments showed that the magnetization of the tips we used was not altered by the dot's stray field nor by the external field. The magnetic field of the tip was calculated analytically.

A $245 \mathrm{~nm} \times 147 \mathrm{~nm}$ elliptical Co dot with a height of 7 $\mathrm{nm}$ was discretized into uniformly magnetized cubes with a sidelength of $7 \mathrm{~nm}$. As a consequence of this discretization, only an approximation to the elliptical dot shape is achieved. The magnetization of each cube $m_{i}$ was assumed constant in magnitude $\left(M_{d}=1.4 \times 10^{6} \mathrm{~A} / \mathrm{m}\right)$ but allowed to rotate. All contributions to the interaction energy (the magnetic fields of the other cubes, exchange interaction, tip's stray field and external field) have been expressed as magnetic fields acting on $m_{i}$. For the micromagnetic simulation, an exchange energy constant of $A_{d}=2 \times 10^{-11} \mathrm{~J} / \mathrm{m}$ has been chosen. ${ }^{14}$ Crystalline anisotropy was neglected to account for the polycrystalline nature of the dot.

In order to perform the simulation, all cubes of the dot were initially magnetized in the $x$ direction. Then the simulation was started. Each step of the simulation consisted of two parts. First, the magnetic fields acting on each cube were computed. To save computing time, the magnetic fields of the cubes were approximated by dipole fields for cubes separated by more than $21 \mathrm{~nm}$ and were neglected for cubes separated by more than $210 \mathrm{~nm}$. Second, the LandauLifshitz-Gilbert equation was solved for each $m_{i}$ using a fourth-order Runge-Kutta method with adaptive time steps. The simulation steps were carried out until a local energy minimum was reached. An indication for the minimum was taken so that the maximum rotation of the $m_{i}$ in a single simulation step was less than $0.1^{\circ}$. Additionally, the energy was computed separately and monitored. 


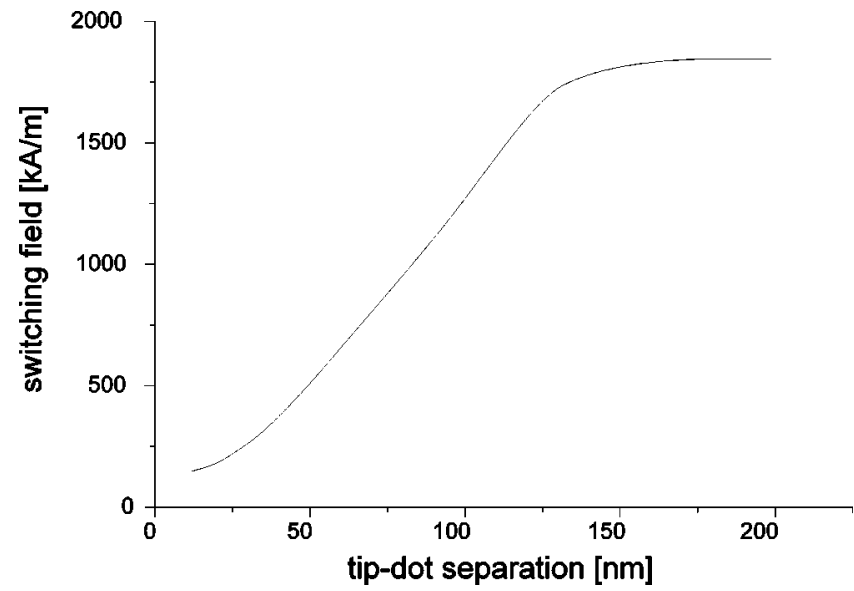

FIG. 7. This diagram shows the minimum field required to reverse the magnetization of a $147 \mathrm{~nm} \times 245 \mathrm{~nm} \times 7 \mathrm{~nm}$ cobalt dot (switching field) as a function of the tip-dot separation.

The tip was positioned $100 \mathrm{~nm}$ right off the center on the long axis and an external field was applied. The simulation was carried out until the energy minimum was reached. The external field was then increased and the simulation of the dot was carried out again. This was repeated until the net magnetization of the dot was pointing in the $(-x)$ direction. This external field was defined as a "switching field" $H_{s w}$ and plotted as a function of the vertical tip-dot separation (Fig. 7). The switching field increases with the tip-dot separation up to about $150 \mathrm{~nm}$. If the tip is moved further away, the switch is only due to the external field and not induced by the tip. After determining the switching field, we visualized different stages of the switching process itself (Fig. 8). The dot has been magnetized in a strong external field to align the magnetization of all cubes along the $x$ direction. Then the external field was turned off and the simulation was started to find the minimum energy for the starting configuration of the switching process [Fig. 8(a)]. Then the tip was positioned $50 \mathrm{~nm}$ above the dot and $100 \mathrm{~nm}$ right off its center. The previously calculated switching field has been applied. In Fig. 8(b) one can see a change of the magnetization along the $(-x)$ direction (upper- right and lower-right part of the dot). Two domain walls and a disturbance on the right can be seen . In Fig. 8(c) vortices show up that subsequentially move out of the dot [Figs. $8(\mathrm{~d})-8(\mathrm{~g})]$ while the area of homogenous magnetization along the $(-x)$ direction increases. In Fig. 8(g), the dot is mostly magnetized in the $(-x)$ direction except the right-hand side where the influence of the tip's stray field is still visible. After the tip is removed and the external field is switched off, the dot stays in a single-domain state [Fig. 8(h)].

Our micromagnetic simulations suggest that the switching process cannot be described by a uniform rotation of all magnetic moments in the dot (Stoner Wohlfarth ${ }^{15}$ ) but by a nucleation and movement of vortices and domain walls caused by the superposition of the tip's stray field and the homogenous external field. This explains why the magnetization of a dot can be switched with lower external fields if the MFM tip is scanning over the dot. A quantitative comparison shows that the switching fields determined by the simulation are about five times higher than those used in the
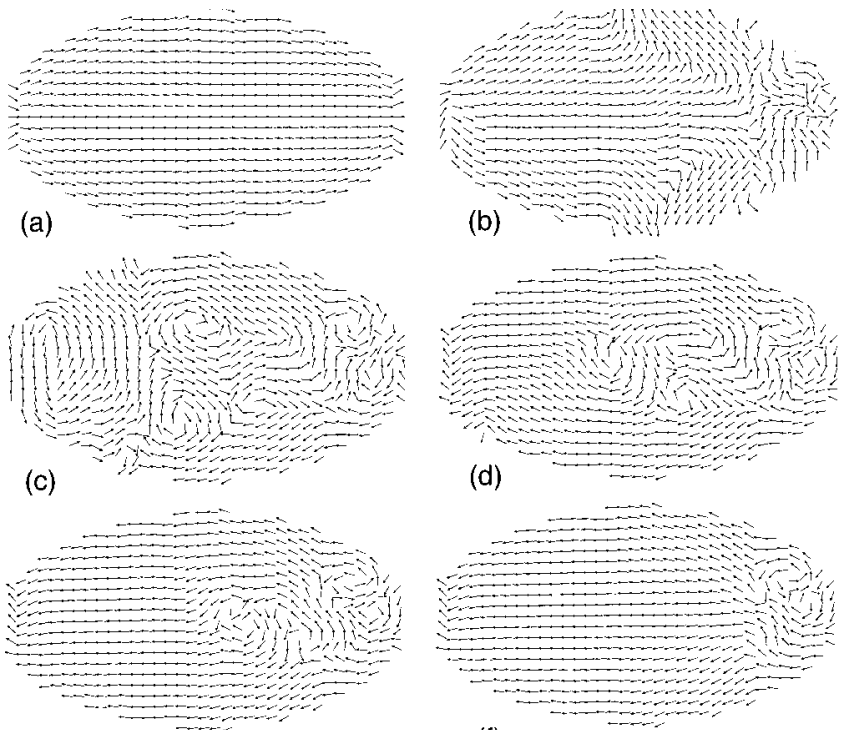

(e)
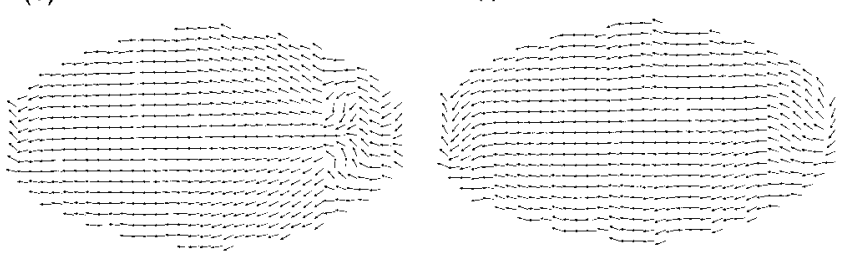

(g)

(h)

FIG. 8. Simulated stages of the magnetization configuration of the dot during the switching process. The arrows represent the $x$ and $y$ components of the dot magnetization at the position marked by the tail of an arrow. (a) the prepared single-domain state of the dot before any field is applied. (b) - (g) intermediate stages of the switching process. The tip is positioned $50 \mathrm{~nm}$ above the surface of the dot $100 \mathrm{~nm}$ toward its right end. An external field of $500 \mathrm{kA} / \mathrm{m}$ is applied in the $(-x)$ direction. (h) Final stage, external field and tip are removed. The magnetization of the dot has switched.

experiment. Further investigation of the internal structure of the dots will probably be necessary to explain this difference.

\section{SUMMARY}

In summary, we have applied magnetic force microscopy to study the magnetic switching behavior of cobalt dots. Dots of diameters below $250 \mathrm{~nm}$ and a height of $7 \mathrm{~nm}$ appeared to be in a single-domain state with the magnetization lying inplane. Elliptical dots are preferentially magnetized parallel to their long axis due to shape anisotropy. By combining an external field with the stray field of the ferromagnetic tip, we changed the magnetization direction of a single dot by $180^{\circ}$. From our micromagnetic simulations we conclude that the switching process is due to a nucleation of domain walls and vortices in the dot. Theoretical simulations also show that the external field that is needed to switch the magnetization of a dot decreases when the magnetic tip is getting closer to this dot.

\section{ACKNOWLEDGMENTS}

Financial support from the BMBF (Grant No. 03N1023A) and the Graduiertenkolleg "Nanostrukturierte Festkörper" is gratefully acknowledged. 
${ }^{1}$ S. Y. Chou, M. Wei, P. R. Krauss, and P. B. Fischer, J. Vac. Sci. Technol. B 12, 3695 (1994).

${ }^{2}$ R. M. H. New, R. F. W. Pease, and R. L. White, J. Magn. Magn. Mater. 155, 140 (1996).

${ }^{3}$ P. D. Ye, D. Weiss, K. von Klitzing, K. Eberl, and H. Nickel, Appl. Phys. Lett. 67, 1441 (1995).

${ }^{4}$ P. D. Ye, D. Weiss, R. R. Gerhardts, M. Seeger, K. von Klitzing, K. Eberl, and H. Nickel, Phys. Rev. Lett. 74, 3013 (1995).

${ }^{5}$ A. M. Barany and H. N. Bertram, IEEE Trans. Magn. 23, 1776 (1987).

${ }^{6}$ C. Kittel, Phys. Rev. 79, 965 (1946).

${ }^{7}$ W. F. Brown, Jr., J. Appl. Phys. 39, 993 (1968).

${ }^{8}$ G. A. Gibson and S. Schultz, J. Appl. Phys. 73, 4516 (1993).
${ }^{9}$ Y. Martin, C. C. Williams, and H. K. Wickramasinghe, J. Appl. Phys. 61, 4723 (1987).

${ }^{10}$ X Löhndorf, A. Wadas, G. Lütjering, D. Weiss, and R. Wiesendanger, Z. Phys. B 101, 1 (1996).

${ }^{11}$ R. Gomez, R. M. H. New, R. F. W. Pease, and R. L. White, J. Appl. Phys. 80, 342 (1996).

${ }^{12}$ NANOSCOPE IIIa, Digital Instruments, Santa Barbara, California.

${ }^{13}$ J. O. Oti, IEEE Trans. Magn. 29, 2359 (1993).

${ }^{14}$ C. Kittel, Rev. Mod. Phys. 21, 541 (1949).

${ }^{15}$ A. Morrish, The Physical Principles of Magnetism (Wiley, New York, 1965). 